\title{
Langzeitverhalten von Talsperren
}

Wasserkraftanlagen sind für die Nutzung durch mehrere Generationen ausgelegt und erreichen damit ein exzellentes Verhältnis von Energieernte zu aufgewendeter Energie für die Errichtung - dies trifft insbesondere für Großkraftwerke zu. In einem gesellschaftlich akzeptierten Ausmaß will es uns auch gelingen, diese erneuerbare Energiequelle umweltverträglich zu nutzen und weiter zu erschließen. Eine Ressourcen schonende, für einen langen Zeitraum ausgelegte Nutzung der Wasserkraft ist deshalb außer Zweifel unter „Nachhaltigkeit“" zu subsumieren und ist in unserer Gesellschaft ein positiv besetzter Begriff.

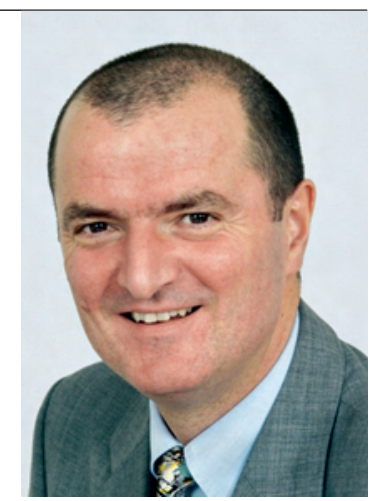

Univ.-Prof. DI Dr. Gerald Zenz

In Österreich bestehen für die Planung, den Bau, den Betrieb und die Überwachung großer wasserbaulicher Anlagen gut etablierte Vorgehensweisen. Auf dem nachweislich positiven Verhalten der bestehenden Bauwerke beruht das Vertrauen der Öffentlichkeit in die Technologie der Wasserkraft. Dieses Vertrauen wird in der Öffentlichkeit durch Berichte und Veranstaltungen gestärkt. Die für dieses Schwerpunktheft der Österreichischen Wasser- und Abfallwirtschaft ausgewählten Beiträge geben mit Bezug auf einen längeren Zeitraum einen Überblick über das Verhalten des Untergrundes, der Wartung von Drainagen, das gute Verhalten des Baustoffes Beton und des gesamten Sperrentragwerks. Aus diesen Erfahrungen lassen sich wieder Rückschlüsse auf Planungskonzepte und Bauweisen ziehen, um Verbesserungen einzuleiten.

Im Rahmen der 2. internationalen Konferenz "Long Term Behaviour of Dams“ (12.-13. Oktober 2009, Technische Universität Graz) wird dem Thema „Langzeitverhalten von Talsperren“ ein darüber hinaus gehendes, weitreichendes Diskussionsforum geboten (www.ltbd09.tugraz.at). Im Rahmen dieser Veranstaltung werden Praktiker und Wissenschafter sowohl das beobachtete als auch das modellierte Verhalten der Bauwerke gemeinsam erörtern, Verbesserungen anregen sowie die klare Kommunikation der Methoden fördern. Dabei ist die internationale Perspektive für die Weitergabe der Erfahrung von besonderer Bedeutung.

Die Nutzung von Talsperren über einen langen Zeitraum erfordert die gesicherte Weitergabe von speziellem Wissen an die Mitarbeiterinnen und Mitarbeiter. Diesbezüglich bieten Universitäten Lehrveranstaltungen an, die durch Kurse von Interessenvertretungen, wie z. B. dem Österreichischen Nationalkomitee für Talsperren (ATCOLD), ergänzt werden. Im Rahmen des 8. ICOLD European Club Symposiums mit dem Titel „Sustainability in a Changing Environment“ in Innsbruck (22. und 23. September 2010, www.iecs2010.tugraz.at), werden diese Themen besprochen. Mit hoher Priorität gilt es, junge Menschen weiterhin für die universitäre Ausbildung zu Technikerlnnen zu begeistern.

Darüber hinaus bietet die Beschäftigung mit bestehenden Anlagen die Möglichkeit, gut bewährte Ingenieurbaukunst zu würdigen und sie für Herausforderungen in der Erhaltung und für den Neubau zu nutzen. Dabei ist die gesamte Anlage mehr als die Summe ihrer Einzelteile, deren manchmal „konstruktive Aspekte" lohnende Aufgaben darstellen, wissenschaftlich begründet zu werden.

\section{Univ.-Prof. DI Dr. Gerald Zenz}

Technische Universität Graz

Institut für Wasserbau und Wasserwirtschaft

Stremayrgasse 10/II

$8010 \mathrm{Graz}$

E-Mail: gerald.zenz@tugraz.at 